\title{
Do pensamento clínico, segundo Foucault, ao resgate do modelo biopsicossocial: uma análise reflexiva
}

\author{
Cleya da Silva Santana CRUZ ${ }^{1}$ \\ Daisy de Rezende Figueiredo FERNANDES ${ }^{2}$ \\ Marcos Luciano Pinheiro PIMENTA ${ }^{3}$ \\ Leida Calegário de OLIVEIRA ${ }^{4}$
}

\author{
${ }^{1}$ Enfermeira, Mestranda do Programa de Pós-Graduação em Saúde, Sociedade e Ambiente da Universidade Federal dos \\ Vales do Jequitinhonha e Mucuri-UFVJM. joaquimcezar@yahoo.com.br \\ ${ }^{2}$ Enfermeira, Doutoranda. Docente do Departamento de Enfermagem da Faculdade de Ciências Biológicas e da Saúde- \\ FCBS/UFVJM. daisygouveia@oi.com.br \\ ${ }^{3}$ Farmacêutico, Doutor. Docente do Departamento de Ciências Básicas /FCBS/UFVJM. marcospimenta2@gmail.com \\ ${ }^{4}$ Bióloga, Doutora. Docente do Departamento de Farmácia /FCBS/UFVJM. leida@ufvjm.edu.br
}

\section{Recebido em: 30/05/2013 - Aprovado em: 12/07/2013 - Disponibilizado em: 15/08/2013}

\section{Resumo:}

Este trabalho faz uma apresentação da passagem da medicina clássica à medicina moderna, tendo como base os estudos de Michel Foucault. Foi descrita inicialmente a medicina clínica sob o ponto de vista foucaultiano, com ênfase na descoberta da medicina anátomoclínica. A passagem da medicina clássica à medicina moderna dá-se em um cenário de nascimento do empirismo e valorização do conhecimento consubstanciado pela anatomia patológica. A partir desse momento a anatomia patológica fundamenta à clínica, havendo mudança na relação entre o objeto que se deve conhecer e aquilo que é cognoscível e desta maneira, uma reformulação do saber médico. A partir da publicação do Relatório Flexner, em 1910, o conceito de saúde passa a basear-se na ausência de doenças e a formação médica ganha uma tendência à especialização. Apesar de vigorar por mais de 100 anos, os novos desafios da saúde colocaram em xeque este modelo flexneriano. Posteriormente, a biomedicina destaca-se no cenário mundial, inserindo-se na prática da medicina. Buscando uma maior efetividade das ações do SUS, hoje no Brasil surge a necessidade da busca do resgate do modelo biopsicossocial e da centralidade na pessoa e na comunidade.

Palavras - chave: Medicina clínica. Educação médica. Medicina clássica. Medicina moderna. Modelo biopsicossocial.

\section{ABSTRACT}

This paper is a presentation about the transition from the classical medicine to the modern medicine, based on Michel Foucault's studies. Clinical medicine was initially described, from Foucault's point of view, with emphasis on the discovery of anatomo-clinical medicine. The transition from classical medicine to modern medicine is given in a scenario of birth of empiricism and enhancement of knowledge embodied by pathology. Thereafter pathological anatomy merges the clinic, with change in the relationship between the object to be known and what is knowable and thus, a redesign of medical knowledge. Since the publication of the Flexner Report in 1910, the concept of health shall be based on the absence of disease and medical education earns a tendency to specialization. Although effective for more than 100 years, new health challenges put the flexnerian model into question. Subsequently, biomedicine stands 
out on the world stage, adhering to the practice of medicine. Seeking greater effectiveness of the Health Care System in Brazil (SUS) arises, nowadays, the need for search and rescue of the biopsychosocial model, and in a person and community centered system.

Keywords: clinical medicine, medical education. classical medicine, modern medicine. Biopsychosocial model.

\section{O NASCIMENTO DA CLÍNICA}

No livro "O Nascimento da Clínica", Michel Foucault (2011) faz um estudo arqueológico do saber médico ocidental, que por meio de uma análise estrutural, relata os caminhos da medicina do século XIX. Foucault investigou a produção do conhecimento da medicina, a transformação operada na estrutura de sua racionalidade e no domínio de sua experiência (SOUZA, 1998). Neste momento, a medicina criticou seu passado e apresentou-se como medicina científica. Diante da análise desta forma de caminhar da medicina tradicional para a moderna, é possível trazer um entendimento do atual paradigma da medicina e dos desafios da busca da valorização da centralidade do cliente e do modelo de atenção biopsicossocial.

Foucault (2011) descreve as modificações e evolução da medicina clássica até a formação da medicina moderna. $\mathrm{Na}$ medicina das espécies as doenças eram classificadas em espécies, e consideradas entidades sem qualquer ligação com o corpo, em que a doença aconteceria quando uma de suas qualidades apresentasse afinidades com o corpo humano. Já com o surgimento da medicina de classificação das doenças, a prática médica acontecia segundo as características visíveis da doença, baseando- se em uma percepção essencial e ordinal, onde apenas o sentido da visão era utilizado.

O fato do médico ter se aproximado do doente trouxe a utilização de outros órgãos dos sentidos como a visão, o tato e a audição e de toda uma semiologia armada (FOUCAULT, 2011). Assim, permitiu-se à medicina penetrar na profundidade do corpo e descobrir a doença. Segundo Queiroz (1986), até esse momento o modelo denominado "medicina ao lado da cama" foi dominante, sendo sua característica principal o fato de que o paciente estar no centro do processo médico, sendo ratado em sua totalidade. Ocorre então uma mudança de posicionamento do modelo médico, passandose ao apogeu da medicina clínica, chamada medicina dos sintomas, em que as doenças eram fenômenos dinâmicos. A soberania do olhar clínico buscava tornar visível tudo o que se encontrava obscuro. A doença apresentavase ao observador do século XVIII, através de sintomas e signos, onde os sintomas eram a primeira transcrição, a primeira camada de uma verdade totalmente exposta ao olhar. Através deles a doença deixava-se transparecer. O signo, por sua vez, anunciava o que ia se passar, fazia a anamnese do que se passou e o diagnóstico do que estava acontecendo no momento. Através do invisível, o signo indicava o que estava mais 
oculto, mais distante. O sintoma era transformado em signo através da intervenção de uma consciência (FOUCAULT, 2011).

A anatomia patológica se tornou uma ciência com o objetivo de conhecer as alterações visíveis que o estado de doença produzia nos órgãos do corpo humano. Isso fez com que a mudança do saber médico do século XVIII gerasse a percepção de algo atrás do visível, além de descobrir a doença na profundidade do corpo (FOUCAULT, 2011).

Através desta visível invisibilidade houve progresso na observação, fidelidade ao que os dados sensíveis podiam revelar, e com isso um crescente empirismo científico dentro da medicina.

Para Foucault (2011), o que modificou com o surgimento da medicina anátomopatológica foi à disposição de saber entre o que se deve conhecer e aquilo que é cognoscível. O acesso do olhar médico ao interior do corpo foi o resultado de uma reformulação do saber e não do nível de conhecimento acumulado.

De fato, antes desse período de reformulação os médicos perguntavam ao doente: "o que há de errado com você?", passando então a perguntar "onde dói". Houve uma reorganização na forma de olhar o doente. A arte de curar indivíduos doentes transforma-se numa disciplina das doenças. $\mathrm{O}$ alvo privilegiado da intervenção médica torna-se, cada vez mais, o corpo individual, o que ajuda a constituir o indivíduo moderno, átomo de um corpo mais amplo (AUGUSTO, 1989).

E é exatamente neste momento, em que o poder do médico cresce exponencialmente, que a saúde substitui a salvação da alma. O poder médico alcançado neste período estende-se aos dias atuais e ocupa-se do orgânico devido a essa tradição secular, sendo limitado à prática cotidiana para lidar com o ser biopsicossocial. Pois, ao lidar com o ser biopsicossocial, a medicina moderna não encontra o terreno anátomo $\mathrm{e}$ fisiopatológico a que foi exercitado a descobrir (FLORA, 2006).

\section{O EMPIRISMO, O SABER MÉDICO E A BIOMEDICINA}

Com o e remodelamento do pensamento médico e com a valorização da atividade clínica, inicia-se a era moderna conhecida como medicina baseada em evidências, em que a prática deve ter como base o exercício do olhar. A atitude do médico foi se cristalizando até chegar ao papel social de um observador neutro, com a atenção voltada para a doença e os seus múltiplos disfarces. Este investigador das pistas é levado a identificar o mal que acomete o doente, podendo incorrer, às vezes, ao erro de esquecer a pessoa por trás da doença (FLORA, 2006).

Nesse paradigma, a relação médico/paciente é coberta por uma nuvem negra que se coloca diante do sujeito cognoscível. De acordo com Souza (1998), a 
transformação da medicina em uma ciência empírica aconteceu por simples aproximação progressiva entre o sujeito e o objeto do conhecimento, tornando, assim, seu conhecimento científico.

Enquanto a saúde era baseada como ausência de doenças, a formação médica se voltou para o tratamento e prevenção das mesmas. Um importante ordenador desse tipo de formação foi $o$ americano Abraham Flexner que, em 1910, publicou o estudo Medical Education in the United States and Canada - "A Report to the Carnegie Foundation for the Advancement of Teaching”, conhecido como Relatório Flexner, que tratava da educação médica. Este relatório exerceu influência nas escolas médicas do Brasil a partir dos anos 50 e gerou profundas implicações para a formação médica e a medicina mundial (PAGLIOSA E DAS ROS, 2008). As bases do Relatório eram de que o estudo da medicina era centrado na doença, sendo que as áreas sociais e o coletivo, não eram considerados na educação médica, apresentando-se o hospital como principal instituição de transmissão de conhecimentos. O modelo de Flexner marcou a passagem da medicina para a biomedicina moderna (KEMP E EDLER, 2004), sendo considerado ainda como uma matriz disciplinar e pedagógica de ensino (ALMEIDA FILHO, 2010).

Não há como negar que o Relatório Flexner expressou um arranjo político no qual as expectativas de mudança no sistema educacional consolidado dependiam de sua estrutura de poder e das expectativas de carreira consagradas pela tradição.

\begin{abstract}
A base desse modelo foi de tal ideário de ensino que se impôs ao resto do mundo no decorrer do século $X X$, operando, de um lado, a ideia de que a qualidade da formação em medicina é igual à capacidade de dominar e de aplicar corretamente os princípios das disciplinas biológicas e da clínica médica e, de outro, em relação à prática médica, propagando o conceito de que ela é proporcional ao tempo de contato que seus agentes tenham tido, e ainda tenham de alguma forma, com laboratórios das ciências biomédicas e com clínicas hospitalares bem equipadas. Esse modelo influenciou a formação médica no Brasil e América Latina e perdeu força a partir da Conferência Internacional de Alma Ata, em 1978, quando ganham força as ideias da Medicina Comunitária, também chamada de Cuidados Primários de Saúde, Medicina Simplificada etc. Bertrand Dawson fez, em 1920, a crítica do modelo flexneriano, e elaborou um relatório propondo a reestruturação dos serviços de saúde que seriam responsáveis por ações preventivas $e$ terapêuticas. (HUBNER E FRANCO, 2007p. 3)
\end{abstract}

Almeida e Filho (2010) apresentam quatro pontos de vista a respeito do relatório Flexner:

1) Conceitual: Com uma perspectiva exclusivamente biologicista de doença, com negação da determinação social da saúde; formação laboratorial no ciclo básico; formação clínica em hospitais; estímulo à disciplinaridade numa abordagem reducionista do conhecimento.

2) Pedagógico: o modelo de ensino preconizado por Flexner é considerado massificador, passivo, 
hospitalocêntrico, individualista e tendente à superespecialização, com efeitos nocivos sobre a formação profissional em saúde.

3) Da prática de saúde: educação superior elitizada, subordinação do ensino à pesquisa, fomento à mercantilização da medicina, privatização da atenção em saúde, controle social da prática pelas corporações profissionais. Do ponto de vista da organização dos serviços de saúde, o Modelo Flexneriano tem sido responsabilizado pela crise de recursos humanos que, em parte, produz crônicos problemas de cobertura, qualidade e gestão do modelo assistencial, inviabilizando a vigência plena de um sistema nacional de saúde integrado, eficiente, justo e equânime em nosso país.

4) Político: implantado no Brasil a partir da Reforma Universitária de 1968, promovida pelo regime militar. Tal modelo de ensino e de prática mostra-se incompatível com o contexto democrático brasileiro e com as necessidades de atenção à saúde de nossa população e dele resultam sérias falhas estruturais do sistema de formação em saúde.

\footnotetext{
A Vigência do Relatório Flexner por mais de 100 anos influenciou a ciência moderna nos currículos das escolas de saúde, o que contribuiu para que, no século $\mathrm{XX}$, a expectativa de vida da população mundial dobrasse. Seu lugar privilegiado foi a
}

universidade, Contudo, os novos desafios da saúde colocaram em xeque o modelo flexneriano de ensino das profissões de saúde. A educação profissional flexneriana não é coerente com os desafios atuais porque é fragmentada, voltada para realidades demográfica e epidemiológica passadas e propiciada por currículos estáticos que não formam graduados para superar os problemas contemporâneos (MENDES, 2012).

\section{A BUSCA DA REINTEGRAÇÃO}

A separação entre medicina Preventiva e Curativa foi criticada por Dawson uma década após a publicação do relatório Flexner. Dawson considerava que a formação médica não deveria dar-se apenas nos hospitais de ensino especializado, pois o médico generalista deveria exercer funções junto ao indivíduo e à comunidade (HUBNER E FRANCO, 2007). Para isso, preconizou a organização do sistema de atenção à saúde em diversos níveis: os serviços domiciliares, os centros de saúde primários, os centros de saúde secundários, os serviços suplementares e os hospitais de ensino. Descreveu as funções de cada nível de atenção e as relações que deveriam existir entre eles, vindo estas descrições a tornarem-se o texto fundante da regionalização dos sistemas de atenção à saúde organizados em bases populacionais, influenciando a organização desses sistemas em vários países do mundo (MENDES, 2012). 


\section{O PARADIGMA BIOPSICOSSOCIAL}

Diante de todo o processo de evolução histórica da medicina e desta nova postura da medicina moderna a partir do século $\mathrm{XX}$, houve a necessidade de reintegração da dimensão psicossocial às práticas de saúde, visando à construção desse novo modelo em contraposição ao modelo biomédico, com a contribuição de diversas áreas do conhecimento (DE MARCO, 2003).

De Marco (2003) explica que o avanço tecnológico na medicina $\mathrm{e} \quad \mathrm{o}$ culto às especializações, embora tenham aumentado os recursos diagnósticos e terapêuticos, contribuíram para criar a ilusão de que seria possível "curar doentes" sem o contato mais próximo com a pessoa que adoece. Segundo o autor, outro complicador é o tipo de sociedade em que vivemos, na qual mais valem as aparências, a velocidade e o lucro, do que o cuidado às pessoas.

Considerando o homem como ser biopsicossocial, há que se considerar outros fatores que estão envolvidos nas causas, manifestações, curso e evolução da saúde e das doenças. É necessário que se atente tanto para o desempenho clínico quanto para as habilidades de comunicação. Segundo De Marco (2003), o modelo biomédico trouxe grandes contribuições, mas trouxe também outro grande problema: o que fazer com a psique e a alma.

No Brasil, atualmente, tem-se investido no fortalecimento da medicina centrada na pessoa, com o modelo biopsicossocial, e o trabalho nas habilidades de comunicação, com destaque ainda para a humanização no atendimento dentro do modelo de atenção à saúde (BRASIL, 2005).

No entanto, Mattos (2005) explica que na visão médica predominante, a noção de medicina social parece sem sentido, pois a doença se reduz a uma perspectiva biológica e a medicina se volta ao indivíduo doente. Para reverter esse quadro é necessário ver a medicina como prática social e também reconhecer os determinantes sociais da doença.

No Brasil, a perspectiva de promover mudança no âmbito do SUS, no modelo assistencial e em suas práticas, tem levado à reformulação e estruturação de novos serviços, sobretudo em investimentos na Estratégia de Saúde da Família. As transformações propostas e implementadas incorporam novos significados à figura do sujeito e a intersubjetividade nas ações preventivas e curativas (FAVORETO, 2005).

\section{MARCOS DA EVOLUÇÃO DA MEDICINA}

As figuras 1 e 2 apresentam, esquematicamente, a trajetória da história da medicina, uma reconstituição do passado da ciência médica, baseando-se nos trabalhos de Diniz (2006), Nedy et al. (2005) e Foucault (2011). O objetivo configura-se em apontar, resumidamente, os principais marcos de evolução, trilhando da medicina clássica à moderna. 
Na Grécia a medicina estava ligada à mitologia e à filosofia e baseava-se na busca de uma causa natural das origens. Aristóteles desenvolvia o pensamento da doutrina dos quatro elementos e a medicina tinha um caráter empírico racional (NEDY et al., 2005).

\section{A medicina de Galeno e Hipócrates} influenciou a escola de medicina da idade média. $\mathrm{O}$ ensino médico consistia no estudo e recitação dos textos hipocráticos e galênicos, não necessariamente associados ao ensino prático supervisionado da profissão. A prática na medicina veio com o estudo da Anatomia Patológica, com Morgagni e Virchow, da Microbiologia, com Koch e Pasteur, e da Fisiologia, com Claude Bernard. O estudo da medicina se modificou para incorporar também os conhecimentos oriundos destas áreas básicas no intuito de embasar a prática (NEDY et al., 2005).

Segundo Diniz (2006), o século XVIII teve a medicina como agente políticoeconômico, por ser instrumento de manutenção e reprodução da força de trabalho. Já no século XX, com a mudança de paradigma do modelo da medicina, esta passa a produzir riquezas.

Com o nascimento da medicina moderna e o surgimento do método científico empírico e da medicina anatomopatológica, houve uma reorganização do doente em um espaço coletivo e homogêneo (FOUCAULT, 1993).
Deve-se ainda destacar a elaboração do Relatório Flexner e a sua influência no processo de formação em medicina, como discutido anteriormente. $\mathrm{O}$ modelo de Flexner passou a ser associado ao rígido ensino médico que privilegiava a formação científica de alto nível, baseado no modelo moderno, estimulando a especialização profissional (NEDY et al., 2005).

Diante da grande especialização da medicina, a partir da Segunda Guerra Mundial, configura-se o modelo biopsicossocial, conhecido como etapa científico social da medicina (DINIZ, 2006). No Cenário mundial ganham ênfase a educação e a prática médica, surgindo duas novas abordagens nesta área, uma voltada para a clínica, a Medicina Baseada em Evidências e outra para o campo pedagógico, a Aprendizagem Baseada em Problemas (NEDY et al., 2005).

A formação do paradigma atual está voltada para a medicina centrada na pessoa e na comunidade, como proposta para uma transformação do método clínico. Esse modelo está assegurado em dois componentes principais: um que se refere ao cuidado da pessoa, com a identificação de suas ideais e emoções a respeito do adoecer e a resposta a elas; e o outro que se relaciona com a identificação de objetivos comuns entre médicos e pacientes sobre a doença e sua abordagem, com o compartilhamento de decisões e responsabilidades (RIBEIRO E AMARAL, 2008). 


\section{Medicina clássica até meados do século XVIII}

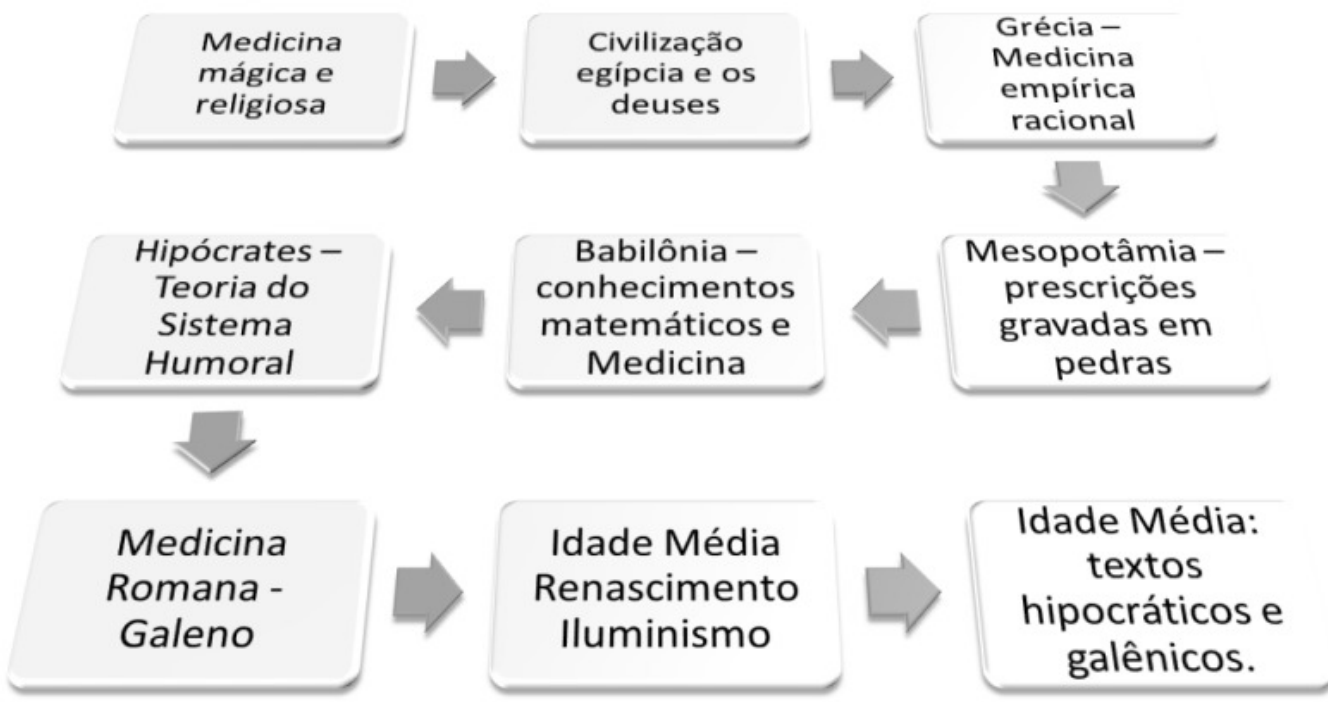

Figura 1: Resumo da evolução da medicina clássica até meados do século XVIII, com ênfase na discussão de Nedy et. al., (2005), Diniz (2006) .

\section{Medicina moderna (final do século XVIII, séculos XIX e XX)}

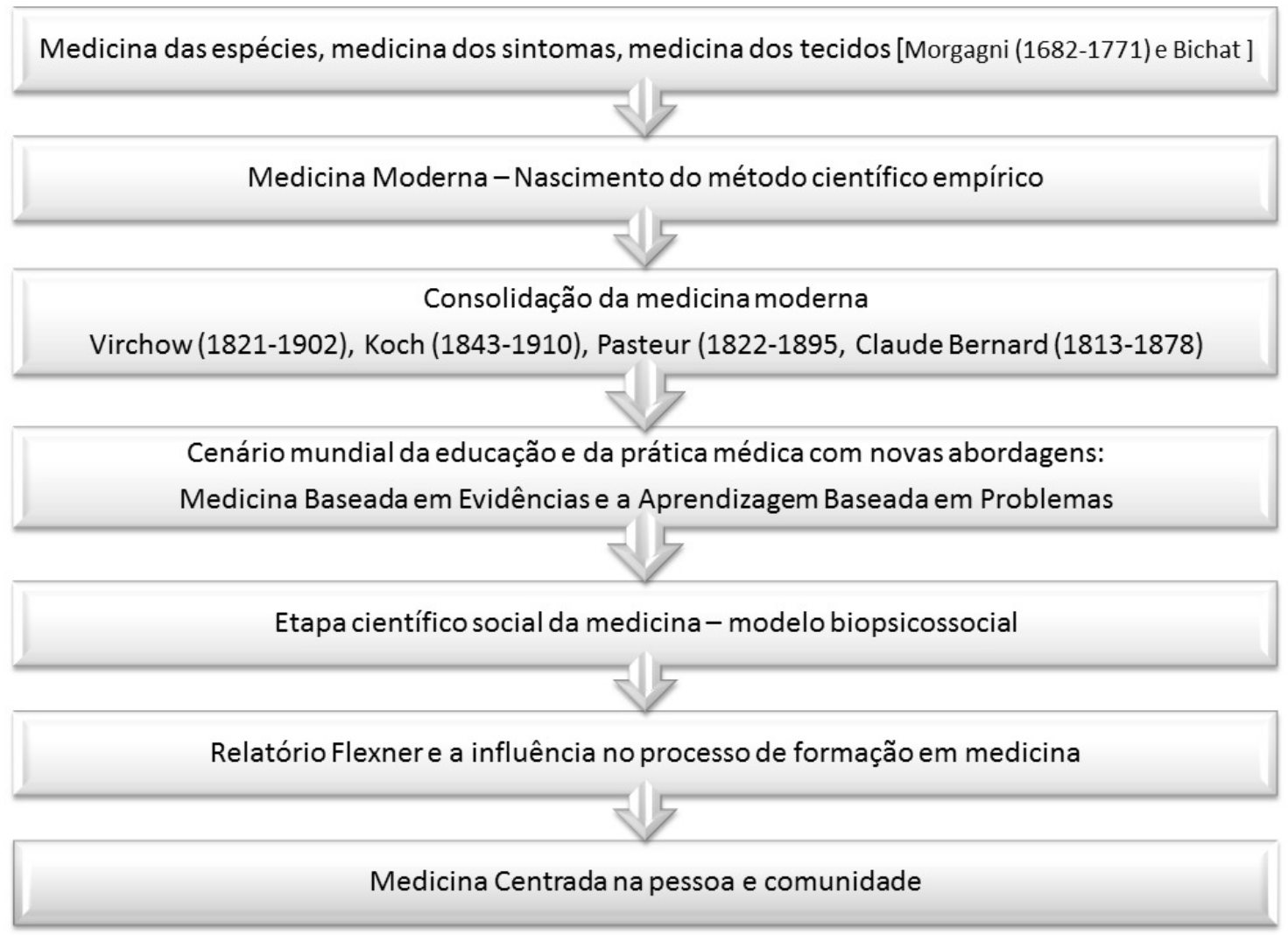

Figura 2: Resumo da evolução da medicina clássica no final do século XVIII, século XIX e século XX, segundo Nedy et. al., (2005) , Diniz (2006) e Foucault (2011). 


\section{CONSIDERAÇÕES FINAIS}

Ao analisarmos a evolução paradigmática da medicina, observamos haver uma tendência de especialização em virtude do grande volume de conhecimento acumulado. permeia todo o pensamento complexo da atualidade.

Durante a idade média tratava-se a doença como expressão de alteração global do organismo em interação com o meio físico e social. Essa integração ficou para trás no século XVIII, quando se estabeleceu um método para se pensar no corpo humano como máquina. Essa ruptura entre saúde e medicina já anunciada pelos tempos culminou no paradigma da biomedicina.
Ocorre com isso uma relação de produção de saber, propiciando também uma tática de poder dentro do serviço de saúde. A medicina moderna privilegia ainda um ponto de objetividade em contramão à subjetividade que

Apesar do modelo biomédico trazer o diagnóstico da doença para além da pessoa, ele trouxe consigo avanços para a ciência médica e, ao mesmo tempo, mostrou-se compatível com o momento capitalista da atualidade. Ainda assim, fez-nos pensar na retomada da articulação da saúde-homem-comunidade e buscar novamente, o modelo em que o traço individual possa buscar o mundo social - o modelo biopsicossocial.

\section{REFERÊNCIAS BIBLIOGRÁFICAS}

ALMEIDA FILHO, N. Reconhecer Flexner: inquérito sobreFAVORETO, C.A.O. A Velha e Renovada clínica dirigida à produção de mitos na educação médica no Brasil. UniversidadeProdução de um Cuidado Integral em Saúde. In: Pinheiro, R. Federal da Bahia, Salvador, Brasil. Cad. Saúde Pública, Rio deMattos, R. A. Cuidado as fronteiras da integralidade. Rio de Janeiro, 26(12):2234-2249, dez, 2010.Janeiro: IMS/UERJ.CEPESC. ABRASCO; 2005.p.205-19.

www.scielosp.org/pdf/csp/v26n12/03.pdf

FLORA, F.A.M. As origens históricas da Clínica e suas AUGUSTO, M.H.O. Natural, racional, social: discussão de umaimplicações sobre a abordagem dos problemas psicológicos na sociabilidade. Tempo Social; Rev. Sociol. USP, S. Paulo, 1(1):prática médica Rev. Bras Med. Fam. e Com Rio de Janeiro, v.2, 247-257, 1.sem. 1989. Comentário crítico da obra de Madeln ${ }^{\circ}$ 7, out 2006. Therezinha Luz. Natural, racional, social: razão médica ewww.rbmfc.org.br/index.php/rbmfc/article/view/56/46 racionalidade científica moderna. Rio de Janeiro, Campus, 1989. http://www.fflch.usp.br/sociologia/temposocial/site/images/st 1993. ories/edicoes/v011/natural.pdf

FOUCAULT, M. Microfísica do Poder. Rio de Janeiro: Graal,

FOUCAULT, M. O Nascimento da Clínica. Rio de BRASIL. Ministério da Saúde. Secretaria de Gestão do Janeiro: Forense Universitária. 2011.

Trabalho e da Educação na Saúde. Departamento de Gestão da Educação na Saúde. A Educação Permanente Entra na Roda. Ministério da Saúde, Brasília, 2005 http://www.google.com.br/\#biw=1024\&bih=427\&sclient=ps y-

HUBNER, L.C.M. and FRANCO, T.B. O Programa Médico de Família de Niterói como Estratégia de Implementação de um Modelo de Atenção que Contemple os Princípios e Diretrizes do SUS. PHYSIS: Rev. Saúde Coletiva, Rio de Janeiro, 17(1):173191, 2007. http://www.scielo.br/scielo.php?pid=S0103-

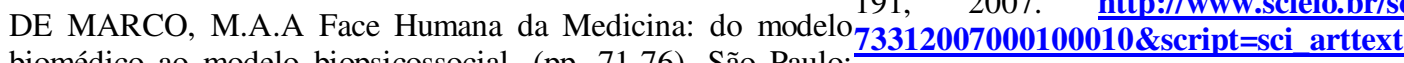
biomédico ao modelo biopsicossocial, (pp. 71-76). São Paulo: Casa do Psicólogo, 2003.DINIZ, D.S.A. Ciência das doenças e a arte de curar: trajetórias da medicina hipocrática. Rio KEMP, A. and EDLER, F.C.: 'Medical reform in Brazil and the Janeiro; 2006. Mestrado [dissertação]. Universidade Federal do Rio de Janeiro www.tesesims.uerj.br/lildbi/docsonline/get.php?id=294

US: a comparison of two rhetorics'. História, Ciências, Saúde - Manguinhos, v. 11 no. 3, pp. 1-16, Sept.-Dec.2004. http://www.scielo.br/pdf/hcsm/v11n3/en_02.pdf 
LUZ, M.T. Natural Racional, Social; Razão Médica e Racionalidade Científica Moderna. Rio de Janeiro: Ed. Campus. 1988.

MATTOS, R.A. Cuidado prudente para uma vida descente. In: Pinheiro, R. Mattos, R. In: Cuidado as fronteiras da integralidade, $2005 \quad$ p. $\quad$ Rio de Janeiro:IMS/UERJ.CEPESC.ABRASCO; 2005.p.205-19.

MENDES, E.V. O cuidado das condições crônicas na atenção primária à saúde: o imperativo da consolidação da estratégia de saúde da família. Organização Pan-Americana da Saúde Organização Mundial da Saúde Conselho Nacional de Secretários de Saúde. Brasília-DF 2012. http://bvsms.saude.gov.br/bvs/publicacoes/cuidado_condico es_atencao_primaria_saude.pdf

NEDY M.B.C. et al. Ensino Médico no Brasil: Origens e Transformações Escola Bahiana de Medicina e Saúde Pública e Faculdade de Medicina da Bahia da UFBA, Gaz méd. Bahia; 75:2(Jul-Dez),

2005.

http://www.medicina.ufba.br/gmbahia/numeros/n_2_2005/G azeta\% 202-2005.pdf

PAGLIOSA, F.. e DA ROS, M.A. Relatório Flexner: Para o Bem e Para o Mal. Revista Brasileira de Educação Médica 32 (4) : 492-499; 2008. www.scielo.br/pdf/rbem/v32n4/v32n4a12.pdf

QUEIROZ, M.S. O paradigma mecanicista da medicina ocidental moderna: uma perspectiva antropológica. Revista de Saúde Pública, São Paulo, 309-317, 1986. http://www.scielo.br/pdf/\% 0D/csp/v9n2/09.pdf

RIBEIRO, M.M.F.; AMARAL, C.F.S. Medicina centrada no paciente e ensino médico: a importância do cuidado com a pessoa e o poder médico. Rev. Bras. Educ. Med., Rio de Janeiro, v. 32, n. 1, mar. 2008. http://www.scielo.br/scielo.php?pid=S0100$\underline{55022008000100012 \& s c r i p t}=$ sci_arttext

RUPP J.C.C. Michel Foucault, a Política do Corpo e a Expansão da Anatomia Moderna PHYSIS - Revista de Saúde Coletiva Vol. $3 . \quad$ Número $2 . \quad 1993$. http://www.scielo.br/pdf/physis/v3n2/01.pdf

SOUZA, A.R.N.D. Formação médica, racionalidade e experiência: O discurso médico e o ensino da clínica. Rio de Janeiro; 1998. Doutorado [tese]. Universidade Federal do Rio de Janeiro.

http://www.psy.med.br/textos/racionalidade/alicia.pdf 